\title{
Energy Conversion and Storage: Synthesis, Mechanism, and Applications of Nanomaterials
}

\author{
Ji Sun Im, ${ }^{1}$ Christopher L. Kitchens, ${ }^{2}$ Jae-Ho Kim, ${ }^{3}$ and Yun Suk Huh ${ }^{4}$ \\ ${ }^{1}$ Division of Green Chemistry and Engineering Research, Korea Research Institute of Chemical Technology (KRICT), \\ Daejeon 305-600, Republic of Korea \\ ${ }^{2}$ Chemical and Biomolecular Engineering, Clemson University, Clemson, SC 29634, USA \\ ${ }^{3}$ Department of Materials Science and Technology, College of Engineering, University of Fukui, Fukui 910-8507, Japan \\ ${ }^{4}$ Department of Biological \& Chemical Engineering, Inha University, Incheon 402-751, Republic of Korea
}

Correspondence should be addressed to Ji Sun Im; jsim@krict.re.kr

Received 26 December 2013; Accepted 26 December 2013; Published 5 February 2014

Copyright (C) 2014 Ji Sun Im et al. This is an open access article distributed under the Creative Commons Attribution License, which permits unrestricted use, distribution, and reproduction in any medium, provided the original work is properly cited.

One of the recent global hot issues is the shortage in energy supply, since the required energy demand is increasing rapidly with limited fuel energy resources. There are numerous attempts to research new abundant and clean energy sources such as hydrogen, solar, natural gas, oil sand, geothermal, wind, and biomass energies as future energy sources. However, this technology has suffered from limited efficiency of generation and storage for industry application. It should be noted that nanotechnology has been a key point for using the above new energy sources based on the unique properties of the nanoscale. Here, we introduced the valuable papers which present the nanotechnology for renewable and green energy.

In the paper entitled "Effects of carbon nanotubes acid treated or annealed and manganese nitrate thermally decomposed on capacitive characteristics of electrochemical capacitors," they discussed how carbon nanotubes (CNTs) can be more effective for an electrode of electrochemical capacitors based on acid and annealing treatments and manganese nitrate thermal decomposition. The optimum conditions for CNTs modification were addressed on high performance of capacitor.

In the paper entitled "Effects of multiwalled carbon nanotube reinforced collagen scaffolds on the osteogenic differentiation of mesenchymal stem cells," they present that multiwalled carbon nanotube- (MWCNT-) collagen scaffolds induced significant increases in alkaline phosphatase (AP) activity and extracellular matrix (ECM) mineralization due to the increased stiffness and strength of the scaffold by entrapping MWCNTs. This technology provides a potential for controlling MSC differentiation using MWCNT-collagen scaffolds.

In the paper entitled "Gold nanorods incorporated cathode for better performance of polymer solar cells," they investigated the effects of gold nanorods incorporation for cathode performance of polymer solar cells. It was revealed that the short circuit current and power conversion efficiency increased on enhancement of absorptivity due to the far-field and nearfield effects of localized surface plasmon resonance induced by the presence of the rods in the interface between the photoactive layer and the metallic rear electrode.

In the paper entitled "Hydrogen storage and release properties of a Cu-added Fe/YSZ redox system," they studied the hydrogen storage and release of iron oxide on redox reaction. It was also discussed that yttria-stabilized zirconia (YSZ) and copper provide the thermal stability and low-temperature activity obtaining $1.8 \mathrm{wt} \%$ storage efficiency after 35 cycles.

In the paper entitled "Hydrogenation of tetralin over supported Ni and Ir catalysts," they present the selective hydrogenation and ring opening (SRO) of tetrahydronaphthalene (tetralin) on nickel and iridium supported catalysts. It was revealed that the $\mathrm{Ir} / \mathrm{SiO}_{2}$ catalyst gave $85 \%$ of tetralin conversion and $75.1 \%$ decalin products selectivity whereas $\mathrm{Ni} / \mathrm{SiO}_{2}$ catalyst showed an unprecedented high catalytic 
performance with $88.3 \%$ of tetralin conversion and $93 \%$ decalin products selectivity.

In the paper entitled "Improvement of lifetime using transition metal-incorporated SAPO-34 catalysts in conversion of dimethyl ether to light olefins," they showed that the lifetime of SAPO-34 nanocatalysts increased by transition metalincorporation. It was presented that the increase in the catalytic lifetime is related to the amount of weak acidic sites, and these sites are increased in number by incorporating transition metals into the SAPO-34 catalyst.

In the paper entitled "Improvement of NO gas sensing properties of polyaniline/MWCNT composite by photocatalytic effect of $\mathrm{TiO}_{2}$," they studied that the sensitivity and response rate of gas sensor were advanced by photocatalytic degradation of $\mathrm{NO}$ by $\mathrm{TiO}_{2}$ in humid condition. The gas sensing mechanism of polyaniline/MWCNT/TiO 2 composite was also discussed that the photo-degraded products such as $\mathrm{HNO}_{2}, \mathrm{NO}_{2}$, and $\mathrm{HNO}_{3}$, which were adsorbed on the PANicoated carbon nanotubes, resulted in the decreased electrical resistance in the p-type semiconductors of carbon nanotube and polyaniline.

In the paper entitled "The effect of nanofluid volume concentration on heat transfer and friction factor inside a horizontal tube," they studied the effects of solid nanoparticle additives $\left(\mathrm{Al}_{2} \mathrm{O}_{3}, \mathrm{TiO}_{2}\right.$, and $\left.\mathrm{SiO}_{2}\right)$ for enhancement of heat transfer and hydrodynamic flow. The simulation was also discussed with Reynolds number and Nusselt number to support their results.

In the paper entitled "Water vapor adsorption capacity of thermally fluorinated carbon molecular sieves for $\mathrm{CO}_{2}$ capture," they present that water vapor adsorption capacity of CMSs increased by fluorination treatment. It was noted that the increased specific surface area and semicovalent C-F group were contributed to higher water vapor adsorption capacity.

In the paper entitled "ZnTe semiconductor-polymer gel composited electrolyte for conversion of solar energy," they studied the effects of ZnTe semiconductor and polymer gel composite as an electrolyte. It was noted that the performance of quasi-solid-state $\mathrm{ZnO}$ DSSCs with different wt\% ZnTeGPE composited electrolytes was the highest efficiency for $0.20 \mathrm{wt} \% \mathrm{ZnTe}$ adding.

\section{Acknowledgments}

We would like to thank the authors for their excellent contributions and patience in assisting us. Finally, the fundamental work of all reviewers on these papers is also very warmly acknowledged.

Ji Sun Im

Christopher L. Kitchens Jae-Ho Kim Yun Suk Huh 

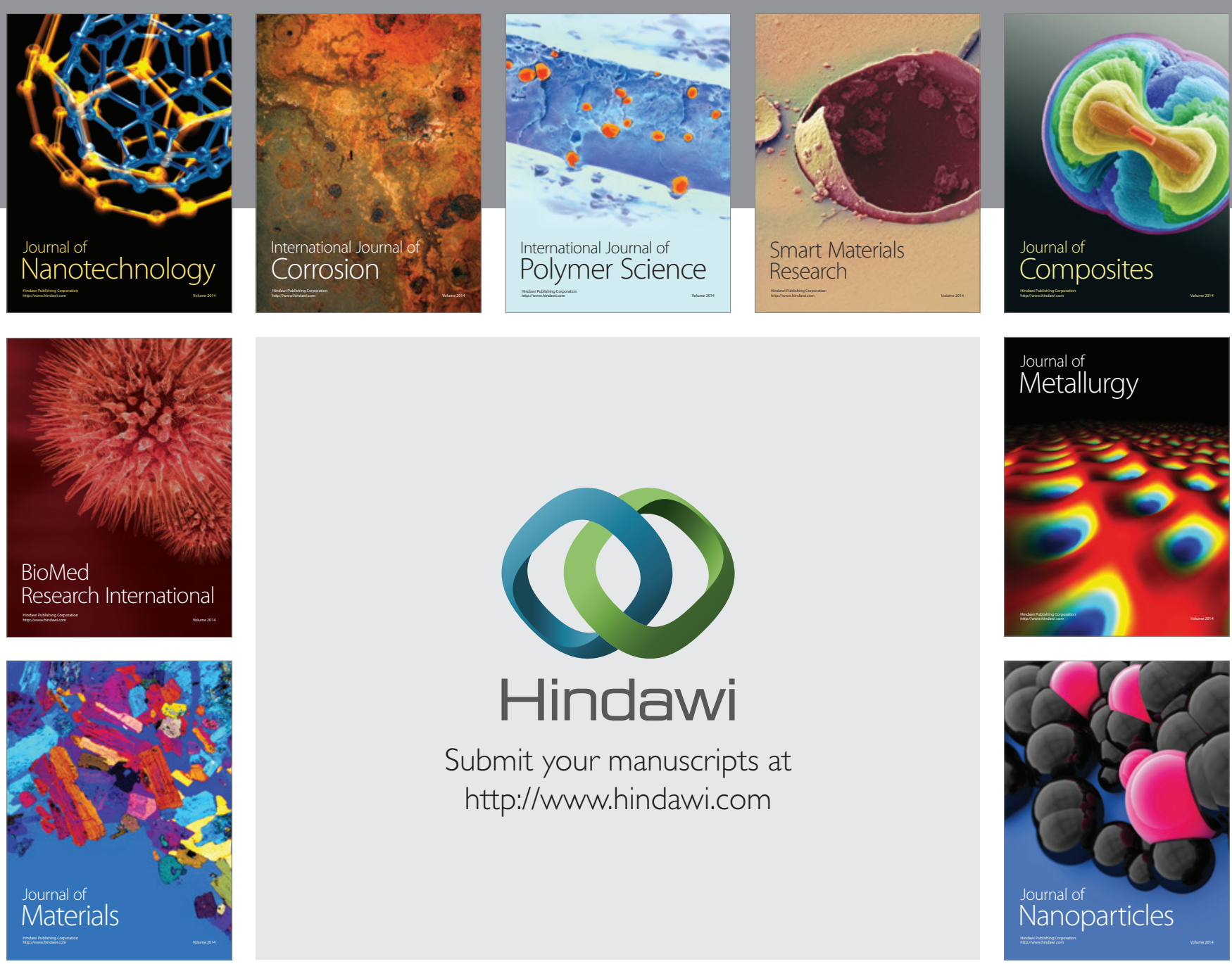

Submit your manuscripts at http://www.hindawi.com
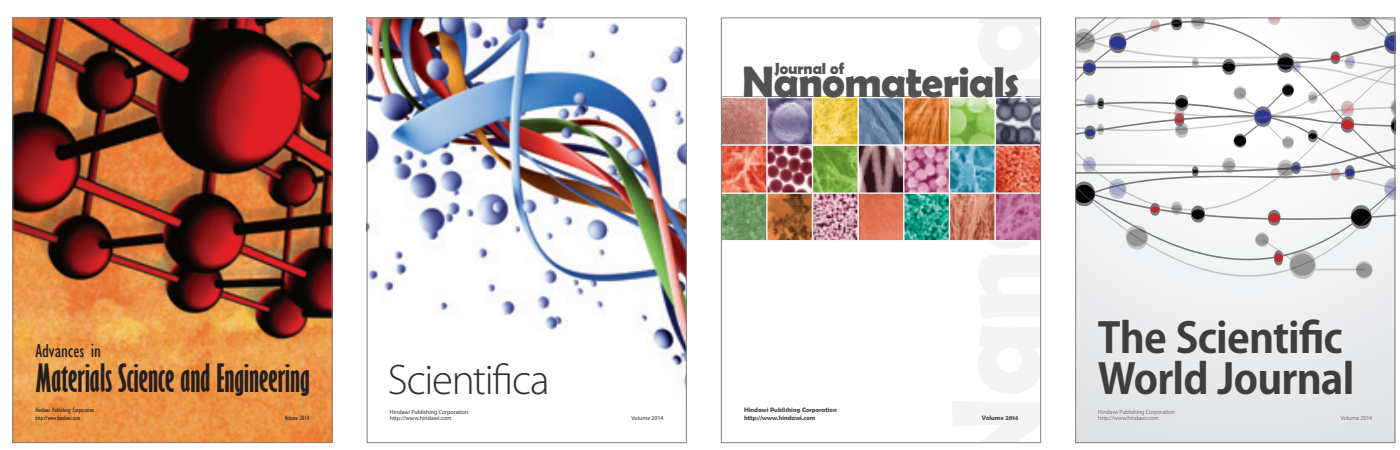

\section{The Scientific World Journal}
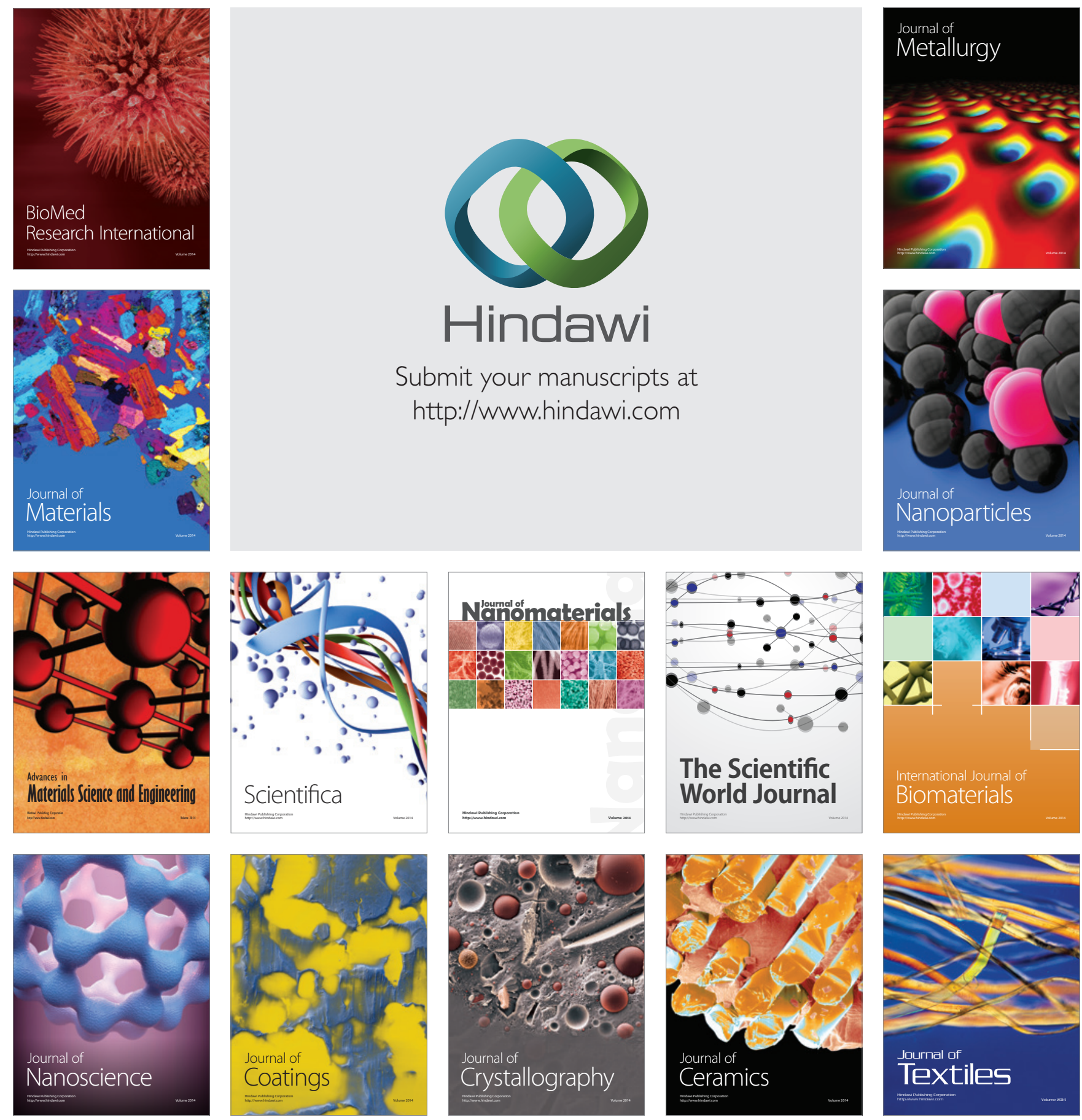